\title{
lutarma
}

\section{Impacto da pandemia pelo novo Coronavírus no perfil de consumo de ansiolíticos e antidepressivos na Atenção Básica do Distrito Federal, Brasil}

\author{
Impact the pandemic by the new Coronavirus on the consumption \\ profile of anxiolytics and antidepressants in the Primary Health Care of \\ the Federal District, Brazil
}

Recebido em: 27/08/2021 Aceito em: 20/12/2021
Kaic Leite MEIRA' ${ }^{1}$ Fernanda Junges de ARAÚJO²; Rafael Cardinali RODRIGUES ${ }^{2}$

${ }^{1}$ Escola Superior de Ciências da Saúde. Setor Médico Hospitalar Norte, Conjunto A Bloco 01 Edificio Fepecs, Asa Norte, CEP 70710-907. Brasilia, DF, Brasil. ${ }^{2}$ Secretaria de Estado de Saúde do Distrito Federal, Administração Central (ADMC). Edifício PO 700 ( $1^{\circ}$ e $2^{\circ}$ andar), Setor de Rádio e TV Norte (SRTVN), 701 Norte, lote D, CEP 70.719-040. Brasilia, DF, Brasil. E-mail:kaic.lmeira@gmail.com

\section{ABSTRACT}

The new Coronavirus pandemic provided an increase in psychosocial vulnerability and the aggravation of preexisting pathologies, such as depression and anxiety. This work aimed to evaluate the impact of the COVID-19 pandemic on the consumption of anxiolytics and antidepressants at UBS 4 in Recanto das Emas - Distrito Federal through an observational, cross-sectional study that analyzed the consumption of anxiolytics and antidepressants in the period between February and August 2019 and 2020, using the average monthly consumption and the number of visits. In addition, information on sex and age was also verified to draw a profile of consumption of these medications. Of the 7 medications evaluated, all showed an increase in consumption in 2020, this being $181.90 \%, 124.36 \%, 325.33 \%, 125 \%, 12.80 \%, 22.18 \%$ and $6.45 \%$ for fluoxetine $20 \mathrm{mg}$, amitriptyline $25 \mathrm{mg}$, imipramine $25 \mathrm{mg}$, clomipramine $75 \mathrm{mg}$, diazepam $5 \mathrm{mg}$, clonazepam $2 \mathrm{mg}$ and clonazepam $2.5 \mathrm{mg} / \mathrm{mL}$ respectively. There was a predominance of females and the population aged between 20 and 59 years as major consumers of these drugs. In general, there was a great impact on the consumption profiles of psychotropic drugs in the period evaluated.

Keywords: mental health; psychotropic drugs; coronavirus; pharmaceutical services.

\section{RESUMO}

A pandemia pelo novo Coronavírus proporcionou o aumento da vulnerabilidade psicossocial bem como o agravo das patologias preexistentes, como depressão e ansiedade. Neste sentindo, o objetivo deste trabalho é avaliar o impacto da pandemia da COVID-19 no consumo de ansiolíticos e antidepressivos na UBS 4 
do Recanto das Emas - Distrito Federal. Trata-se de um estudo observacional, com corte transversal, que analisou o consumo de ansiolíticos e antidepressivos no período entre fevereiro a agosto de 2019 e 2020 , utilizando o consumo médio mensal e o número de atendimentos. Além disso, verificou-se também as informações de sexo e idade para traçar um perfil de consumo destes medicamentos. Dos 7 medicamentos avaliados, todos apresentaram um aumento no consumo em 2020, sendo este de 181,90\%, 124,36\%, $325,33 \%, 125 \%, 12,80 \%, 22,18 \%$ e $6,45 \%$ para a fluoxetina $20 \mathrm{mg}$, amitriptilina $25 \mathrm{mg}$, Imipramina $25 \mathrm{mg}$, clomipramina $75 \mathrm{mg}$, diazepam $5 \mathrm{mg}$, clonazepam $2 \mathrm{mg}$ e clonazepam 2,5 mg/mL respectivamente. Com relação ao perfil encontrado, houve uma predominância do gênero feminino e da população com idade entre 20 a 59 anos, como consumidores majoritários desses medicamentos. De maneira geral, foi observado um grande impacto nos perfis de consumo dos psicotrópicos no período de tempo avaliado.

Palavras-chave: saúde mental; psicotrópicos; coronavírus; assistência farmacêutica.

\section{INTRODUÇÃO}

Em dezembro de 2019, múltiplos casos de pneumonia, oriunda de um agente etiológico desconhecido, começaram a surgir em Wuhan, China (1). Inicialmente, a partir de técnicas de sequenciamento genômico, houve a demonstração de que essa pneumonia se tratava de uma doença, denominada COVID-19, que possui como causador o SARS-CoV-2, um novo tipo de Coronavírus. Essa enfermidade possui uma elevada transmissibilidade e atingiu aproximadamente 72 países no início de março de 2020 (2-4), o que levou a Organização Mundial de Saúde (OMS) decretar estado de pandemia (5).

A pandemia da COVID-19 já atingiu mais 178 milhões de pessoas em todo o mundo (6). No Brasil, até dezembro de 2021, havia mais de treze milhões de casos positivos e mais de seiscentos mil óbitos (7). Diante da rápida disseminação do contágio, da intensa elevação do número de mortos, do iminente colapso nos sistemas de saúde e também funerário, são esperadas complicações para a saúde mental de toda a sociedade (8).

Em situações anteriores de pandemias e endemias, foi observado, além da rápida disseminação da doença, um agravo à saúde mental da população, uma vez que há uma maior quantidade de pessoas afetadas psicologicamente do que as que são acometidas pela doença pandêmica $(9,10)$. Isso, na maioria das vezes, é devido às incertezas acerca da forma de controle e do tempo de duração deste estado de emergência $(11,12)$. Por isso, é comum uma elevação direta da vulnerabilidade psicossocial, principalmente daqueles que apresentam maior dificuldade em reestabelecer meios de subsistência prejudicados pela pandemia (13). Diante deste cenário, é observado o desenvolvimento de sintomas obsessivos-compulsivos, em relação às práticas de higienização, bem como a instalação de um quadro generalizado de ansiedade $(11,12)$. Além do mais, a disseminação de informações equivocadas com relação a infecção, medidas de proteção e tratamento, associadas com a incompreensão da população acerca das medidas sanitárias, contribui ainda mais para o agravo à saúde mental (14).

Dentre as diversas estratégias utilizadas para o manejo das psicopatologias decorrentes da pandemia, há a utilização de psicofármacos, medicamentos que atuam diretamente no Sistema Nervoso Central (15). Na Atenção Primária à Saúde (APS), foi observado o consumo mais frequente destes medicamentos, principalmente dos antidepressivos e ansiolíticos (16). No entanto, o uso não racional desses medicamentos pode resultar em manifestação de efeitos adversos graves, como redução da capacidade motora, insuficiência respiratória e desenvolvimento do fenômeno de dependência (17). Além disso, o aumento desassistido no consumo desses medicamentos implica na elevação dos custos em saúde, uma vez que as hospitalizações e a aquisição de outros fármacos, ainda mais onerosos, são alternativas para o manejo das complicações oriundas do uso não racional dos psicofármacos $(18,19)$. Nesse sentido, o objetivo deste trabalho foi analisar o 
impacto da pandemia pelo novo Coronavírus no perfil de consumo de ansiolíticos e antidepressivos na Unidade Básica de Saúde no 4 do Recanto das Emas, Distrito Federal, Brasil.

\section{MÉTODO}

A pesquisa se trata de um estudo observacional, com corte transversal, que analisou o impacto da pandemia no perfil de consumo de ansiolíticos e antidepressivos na UBS 4 do Recantos das Emas, DF.

O presente estudo foi aprovado pelo Comitê de Ética em Pesquisa da Fundação de Ensino e Pesquisa em Ciências da Saúde (FEPECS) sob o número do parecer 4.388.852.

A análise se deu por meio da comparação do Consumo Médio Mensal (CMM) e do número médio de atendimentos referentes ao período entre fevereiro a agosto de 2019, com os mesmos indicadores referentes ao mesmo intervalo de tempo no ano de 2020. Além disso, foram verificados os dados com relação ao gênero e idade dos usuários para traçar um perfil de consumo.

O CMM foi determinado em ambos os períodos analisados para cada medicamento, por meio da média aritmética dos consumos em cada mês referente ao intervalo de tempo analisado. Os dados utilizados para este cálculo foram obtidos via software Alphalinc ${ }^{\circledR} 2014$ versão 1.70, ferramenta de gestão da Assistência Farmacêutica utilizada pela Secretaria de Estado de Saúde (SES) do DF (20). O número médio de atendimentos mensais dos medicamentos avaliados também foi obtido por meio do mesmo software considerando o mesmo intervalo de tempo, tanto em 2019 quanto em 2020. As informações referentes ao gênero e idade também foram obtidas pelo sistema de gestão da SES-DF, levando-se em consideração o mesmo período de tempo utilizado para estudo.

Foram incluídos na análise os medicamentos do componente básico da Assistência Farmacêutica contidos na Relação Distrital de Medicamentos Essenciais do Distrito Federal (REME) (21), utilizados para tratamento de doenças relacionadas à saúde mental. Os medicamentos que fizeram parte do estudo foram agrupados conforme a classificação farmacológica da Organização Mundial da Saúde (22), sendo eles:
- Antidepressivos: Fluoxetina 20mg, Amitriptilina 25mg, Clomipramina 75mg, Imipramina $25 \mathrm{mg}$

- Ansiolíticos benzodiapínicos: Diazepam $5 \mathrm{mg}$ e Clonazepam $2 \mathrm{mg}$ e Clonazepam 2,5mg/mL

Foram excluídos do estudo os medicamentos que tiveram um abastecimento irregular ou que tiveram seu estoque zerado no período de tempo analisado:

- Clomipramina 25mg, Nitrazepam 10mg, Nortriptilina $25 \mathrm{mg}$ e Nortriptilina $50 \mathrm{mg}$.

Os dados foram organizados e analisados utilizando o programa Microsoft ${ }^{\circledR}$ Excel versão 2019.

\section{RESULTADOS E DISCUSSÃO}

Na Tabela 1, está o consumo médio mensal de comprimidos, cápsulas ou frascos dos antidepressivos avaliados. Houve um aumento de 181,90\%, $124,36 \%, 325,33 \%$ e $125 \%$ no uso de Fluoxetina $20 \mathrm{mg}$, Amitriptilina $25 \mathrm{mg}$, Imipramina $25 \mathrm{mg}$ e Clomipramina $75 \mathrm{mg}$ respectivamente. Com relação aos ansiolíticos, houve um aumento no consumo tanto para o Diazepam $5 \mathrm{mg}$ quanto para Clonazepam $2 \mathrm{mg}$ e $2,5 \mathrm{mg} / \mathrm{mL}$, sendo estes de $12,80 \%, 22,18 \%$ e $6,45 \%$ nessa ordem (Tabela 1). Além disso, o uso de antidepressivos foi bem superior ao de ansiolíticos tanto em 2019 quanto em 2020 (Tabela 2).

Tabela 1. Consumo Médio Mensal (CMM) de medicamentos com ação no Sistema Nervoso Central, entre pacientes do Recanto das Emas, Distrito Federal, Brasil, nos períodos fevereiro a agosto de 2019 e fevereiro a agosto de 2020 .

\begin{tabular}{|l|l|l|}
\hline \multicolumn{1}{|c|}{ ANTIDEPRESSIVOS } & \multicolumn{1}{|c|}{ CMM 2019} & CMM 2020 \\
\hline Fluoxetina $20 \mathrm{mg}$ & $15470 \mathrm{cap}$ & $43610 \mathrm{cap}$ \\
\hline Amitriptilina $25 \mathrm{mg}$ & $8540 \mathrm{crp}$ & $19160 \mathrm{crp}$ \\
\hline Imipramina $25 \mathrm{mg}$ & $1500 \mathrm{crp}$ & $6380 \mathrm{crp}$ \\
\hline Clomipramina $75 \mathrm{mg}$ & $80 \mathrm{crp}$ & $420 \mathrm{crp}$ \\
\hline ANSIOLÍTICOS & CMM 2019 & CMM 2020 \\
\hline Diazepam 5 mg & $1720 \mathrm{crp}$ & $1941 \mathrm{crp}$ \\
\hline Clonazepam 2 mg & $2930 \mathrm{crp}$ * & $3580 \mathrm{crp}$ \\
\hline Clonazepam 2,5 mg/mL & $31 \mathrm{fr}$ & $33 \mathrm{fr}$ \\
\hline
\end{tabular}

Fonte: Alphalinc ${ }^{\circledR}$. cap: cápsulas; crp: comprimidos; fr: frascos. 
Tabela 2. Consumo de psicotrópicos entre pacientes do Recanto das Emas, Distrito Federal, Brasil, nos períodos fevereiro a agosto de 2019 e fevereiro a agosto de 2020 .

\begin{tabular}{|l|c|c|}
\hline Consumo de Psicotrópicos* & 2019 & 2020 \\
\hline Antidepressivos & 25590 cap e crp & 69570 cap e crp \\
\hline Ansiolíticos & 4681 crp e fr & 3793 crp e fr \\
\hline
\end{tabular}

Fonte: Alphalinc ${ }^{\circledast}$. cap: cápsulas; crp: comprimidos; fr: frascos. *calculado por meio da fórmula peso (kg) / altura ${ }^{2}$ (metros) e classificado de acordo com as Diretrizes Brasileira de Obesidade em: normal (IMC $\leq 24,99 \mathrm{~kg} / \mathrm{m} 2$ ), sobrepeso (IMC $25-29,9 \mathrm{~kg} / \mathrm{m} 2$ ) e obeso (IMC $\geq 30 \mathrm{~kg} / \mathrm{m} 2$ ).

Com relação aos números de atendimentos na farmácia, foi observada, de maneira geral, uma pequena variação. Para antidepressivos, houve um pequeno aumento para ambos os gêneros, sendo estes de 7,14\% para o gênero feminino e $28,57 \%$ para o masculino (Tabela 3 ). Com relação aos ansiolíticos, ocorreu uma redução no atendimento das mulheres e um aumento dos homens, sendo este de $17,08 \%$ e $15,12 \%$, respectivamente (Tabela 3).

Avaliando ambas as classes farmacológicas, as mulheres representaram majoritariamente o público de consumo dos medicamentos antidepressivos e ansiolíticos nos períodos do estudo, com percentuais de consumo bem semelhantes (Figura 1). Além disso, com relação a idade, se que não houve uma alteração no padrão de consumo, mantendo a população entre 20 a 59 anos como predominante na utilização dos medicamentos avaliados (Figura 1).

Tabela 3. Número de atendimentos na farmácia do Recanto das Emas, Distrito Federal, Brasil, nos períodos fevereiro a agosto de 2019 e fevereiro a agosto de 2020.

\begin{tabular}{|l|c|c|}
\hline \multicolumn{1}{|c|}{ ANTIDEPRESSIVOS } & 2019 & 2020 \\
\hline Masculino & 350 & 450 \\
\hline Feminino & 1736 & 1860 \\
\hline \multicolumn{1}{|c|}{ ANSIOLÍtICOS } & 2019 & 2020 \\
\hline Masculino & 205 & 236 \\
\hline Feminino & 480 & 398 \\
\hline
\end{tabular}

Fonte: Dados do Alphalinc ${ }^{\circledR}$.
Figura 1. Atendimentos na farmácia do Recanto das Emas, Distrito Federal, Brasil, nos períodos fevereiro a agosto de 2019 e fevereiro a agosto de 2020, quando ao gênero e idade dos pacientes.

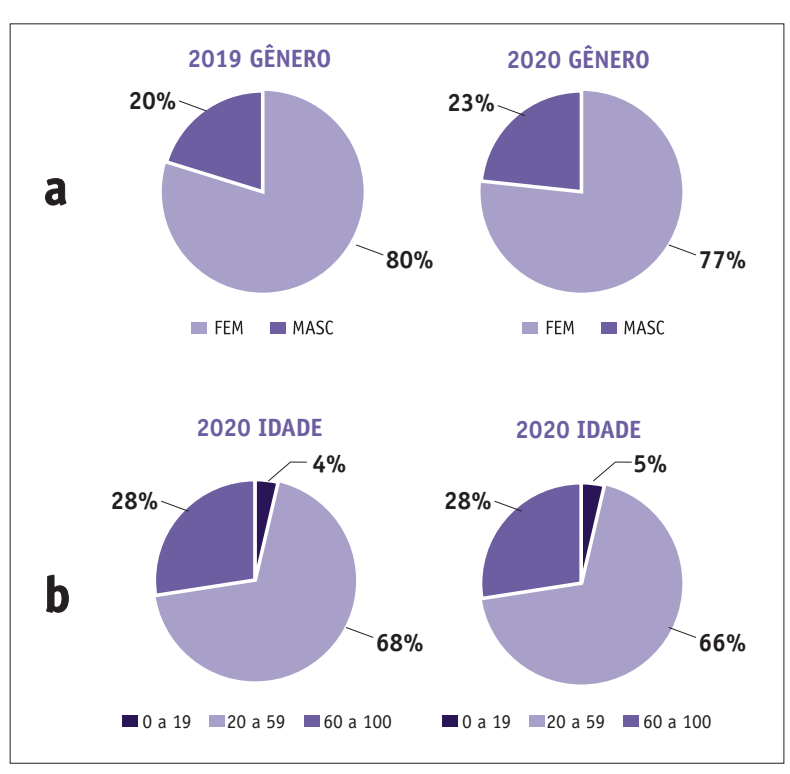

Fonte: Dados do Alphalinc ${ }^{\circledR}$.

De maneira geral, houve um aumento no consumo médio mensal dos medicamentos avaliados. Com relação ao valor do desvio padrão, deve ser considerada a variabilidade no consumo dos itens analisados, uma vez que a quantidade de atendimentos é bem flexível. Além disso, os medicamentos antidepressivos devem ser destacados, pelo seu aumento expressivo. Um dos fatores que podem ter influenciado neste resultado, é o fato da pandemia pelo COVID-19 proporcionar, dentre outros agravos, o desenvolvimento de depressão ou a piora desse transtorno mental pré-existente, uma vez que, na pandemia, há uma redução das interações sociais, que em conjunto com a solidão, torna-se importante fator de risco para a manifestação desta doença $(23,24)$.

Outro ponto que também deve ser levado em consideração, é a assistência à saúde desses usuários. Apesar de ter sido observado o aumento do consumo dos medicamentos avaliados, houve uma reorganização na carteira de serviços da Atenção Primária à Saúde (APS), em decorrência da pandemia, considerando que o acolhimento inicial das síndromes gripais ocorre neste nível de atenção $(25,26)$. Dessa forma, os usuários 
com condições de saúde crônicas, incluindo a depressão e ansiedade, que necessitavam de um acompanhamento longitudinal, possivelmente ficaram sem esta assistência, uma vez que a APS, como organizadora do cuidado, estava sobrecarregada com o COVID-19.

Os resultados indicaram o maior consumo de antidepressivos e ansiolíticos por mulheres. A dupla jornada deste público, cuidando também da família e do lar, em conjunto com o acúmulo de funções das atividades profissionais, em decorrência da maior inserção da mulher no mercado de trabalho, podem ter contribuído para ampliar as demandas de saúde mental no gênero feminino $(27,28)$. Além disso, há uma associação direta entre a inserção das mulheres no mercado de trabalho e a qualidade de vida relacionada à saúde, sendo os piores resultados encontrados na situação em que a mulher apenas assume o papel de dona de casa, principalmente com relação a saúde mental, muito comum na realidade observada. Cabe ressaltar também que episódios estressantes da vida podem influenciar em uma maior morbidade psíquica, vez que há o aumento do sofrimento e angustia $(28,29)$.

$\mathrm{O}$ uso de psicofármacos aumenta conforme a faixa etária dos indivíduos $(30,31)$. No entanto, neste estudo a maior porcentagem de utilização ficou concentrada na população entre 20 a 59 anos. Uma possível justificativa seria o impacto do isolamento social, aliado à crescente taxa de desemprego do País, que se agravou com a pandemia do COVID-19, pois este grupo representa grande parcela da população economicamente ativa. Além disso, o próprio medo de contaminar seus familiares, já se constitui um importante fator contribuinte para o agravo à saúde mental (10).

É incontestável o papel da APS na oferta de cuidados em saúde mental, levando-se em con- sideração os atributos do acesso, longitudinalidade, coordenação do cuidado e integralidade. Além disso, a oferta do cuidado no primeiro nível de atenção tem repercutido melhor na qualidade de vida do usuário do que no especializado, visto que não há uma fragmentação do indivíduo, correlacionando a saúde física com mental. No entanto, a operacionalização destes atributos está comprometida diante da desatenção do atual governo, que enfraquece o modelo de Estratégia de Saúde da Família, base da APS, e levanta dúvidas com relação a continuidade do Núcleo de Apoio à Saúde da Família $(23,32)$.

É importante considerar algumas limitações do estudo. A quantidade máxima que poderia ser dispensada de psicotrópicos em 2019 foi alterada em 2020, para 180 dias (33), sendo isso um fator que pode ter influenciado no resultado observado. Outro fator limitante está relacionado a farmacoeconomia e impacto financeiro desse aumento nos custos gerados para a APS, principalmente diante de uma política nacional de subfinanciamento deste modelo.

\section{CONCLUSÃO}

De maneira geral, a pandemia pelo novo Coronavírus acarretou em profundas mudanças em toda a sociedade. No que se refere ao perfil de consumo dos psicotrópicos na UBS 4 do Recanto das Emas, houve uma variação. No entanto as características de gênero e idade se mantiveram, sendo as mulheres e a população entre 20 a 59 anos, consumidores majoritários de psicotrópicos tanto em 2019 quanto em 2020. Contudo, destaca-se a necessidade da realização de outros estudos que investiguem melhor a real causa do aumento observado, uma vez que há a possiblidade de se ter outros fatores envolvidos, além da pandemia de COVID-19. 
1. Madabhavi I, Sarkar M, Kadakol N. CoviD-19: A review. Monaldi Arch Chest Dis. 2020;90(2):248-258. DOI: 10.4081/monaldi.2020.1298

2. Li H, Liu SM, Yu XH, Tang SL, Tang CK. Coronavirus disease 2019 (COVID-19): current status and future perspectives. Int J Antimicrob Agents. 2020;55(5):105951. DOI: 10.1016/j.ijantimicag.2020.105951

3. Li Q, Guan X, Wu P, Wang X, Lei Zhou, Yeqing Tong, Ren R, Leung KSM, Lau EHY, Wong JY, Xing X, Xiang N, Wu Y, Li C, Chen Q, Li D, Liu T, Zhao J, Man Liu M, Tu W, Chen C, Jin L, Yang R, Wang Q, Zhou S, Wang R, Liu H, Luo Y, Liu Y, Shao G, Li H, Tao Z, Yang Y, Deng Z, Liu B, Ma Z, Zhang Y, Shi G, Lam TTY, Wu JT, Gao GF, Cowling BJ, Yang B, Leung GM, Feng Z. Early transmission dynamics in Wuhan, China, of novel coronavirus-infected pneumonia. N Engl J Med. 2020;382(13):1199-11207. DOI: 0.1056/ NEJMoa2001316

4. Xu X, Chen P, Wang J, Feng J, Zhou H, Li X, Zhong W, Hao P. Evolution of the novel coronavirus from the ongoing Wuhan outbreak and modeling of its spike protein for risk of human transmission. Sci China Life Sci. 2020;63(3):457-460. DOI: 10.1007/s11427-02-1637-5

5. WHO. Coronavirus disease 2019 Situation Report 51 11th March 2020. World Health Organization. 2020;2019:2633. Disponível em: https://www.who.int/emergencies/diseases/ novel-coronavirus-2019 Acesso em 24 de abril de 2021

6. WHO. Coronavirus (COVID-19) Dashboard. World Health Organization. Disponível em: https:/covid19.who.int/ Acesso em 23 de abril de 2021.

7. BRASIL. Ministério da Saúde, Secretaria de Vigilância em Saúde. Boletim epidemiológico $n^{\circ} 59$. Brasília: Ministério da Saúde. 2021.

8. Faro A, Bahiano MA, Nakano TC, Reis C, Silva BFP, VittiLS. COVID-19 e saúde mental: a emergência do cuidado. Estud Psicol. 2020;37. DOI: 10.1590/1982-0275202037e200074

9. Lima RC. Covid-19 no Brasil : impactos na saúde mental. 2020;30(2):1-10. DOI: 10.1590/S0103-73312020300214

10. Ornell F, Schuch JB, Sordi AO, Henrique F, Kessler P. "'Pandemic fear"' and COVID-19 : mental health burden and strategies. 2020;42(3):232-235. DOI: 10.1590/15164446-2020-0008

11. Schmidt B, Crepaldi MA, Bolze SDA, Neiva-Silva L, Demenech LM. Saúde mental e intervenções psicológicas diante da pandemia do novo coronavírus (COVID-19). Estud Psicol. 2020;37:1-13. DOI: 10.1590/1982$0275202037 \mathrm{e} 200063$

12. Zandifar A, Badrfam R. Iranian mental health during the COVID-19 epidemic. Asian J Psychiatr. 2020;51:101990. DOI: 10.1016/j.ajp.2020.101990
13. OPAS. Proteção da Saúde Mental em Situações de Epidemias. Organização Pan-Americana de Saúde. 1-26, 2009.

14. Bao Y, Sun Y, Meng S, Shi J, Lu L. 2019-nCoV epidemic: address mental health care to empower society. Lancet. 2020;395(10224):e37-e38. DOI: 10.1016/S01406736(20)30309-3

15. BRASIL. Ministério da Saúde. Saúde Mental. Cadernos de Atenção Básica (34). Brasília, DF: Ministério da Saúde; 2013.

16. Santos MER, Neves NCV, Almeida JCS, Amparo TR, Piau $\mathrm{AV}$, Dôres RGR. Consumo de fármacos psicotrópicos em uma Farmácia Básica de Congonhas, Minas Gerais, Brasil. Infarma. 2019;285-292. DOI: 10.14450/2318-9312.v31. e4.a2019.pp285-292

17. Gomez SG. Uso de psicofármacos e as ações da Atenção Básica: uma proposta de intervenção no município de Astorga , Paraná. 2018. 29 p. Monogradia (Especialização em Multiprofissional em Atenção Básica) Departamento de Saúde Pública, Universidade Federal de Santa Catarina.

18. Moura D, Pinto J, Martins P, Arruda Pedrosa K, Carneiro M. Uso Abusivo De Psicotrópicos Pela Demanda Da Estratégia Saúde Da Família: Revisão Integrativa Da Literatura. SANARE-Rev Políticas Públicas. 2017;15(2):136-144.

19. Santos GAS, Boing AC. Hospitalizations and deaths from drug poisoning and adverse reactions in Brazil: An analysis from 2000 to 2014. Cad Saude Publica. 2018;34(6):1-14. DOI: 10.1590/0102-311X00100917

20. BRASIL. Assistência Farmacêutica: instruções técnicas para a sua organização. Brasília: Ministério da Saúde, 2002.

21. SES-DF. Relação de Medicamentos do Distrito Federal 2021. Brasília: Secretaria de Estado de Saúde, $2^{\circ}$ ed., 2021.

22. WHO. Collaborating Centre for Drug Statistics Methodology, Guidelines for ATC classification and DDD assignment. World Health Organization 2021;148:148-162.

23. Nabuco G., Oliveira MHPP., Afonso MPD. The impact of COVID-19 pandemic on mental health : what is the role of Primary Health Care ? Rev Bras Med Família Comunidade. 2020;15(42):1-11. DOI: 10.5712/rbmfc15(42)2532

24. Fiorillo A, Gorwood P. The consequences of the COVID-19 pandemic on mental health and implications for clinical practice. Eur Psych. 2020;DOI: 10.1192/j.eurpsy.2020.35

25. SES-DF. Nota Técnica No 01/2020 SES/SAIS/COAPS - COVID-19 Versão 4. Diretrizes de Apoio Clínico e Organizacional para a APS no enfrentamento à COVID-19. Brasília: Secretaria de Estado de Saúde, 2020

26. SES-DF. Circular n. ${ }^{\circ}$ 4/2020 - SES/SAIS/COAPS/DESF. Recomendações para adequação das atividades dos Agentes Comunitários de Saúde (ACS) frente à pandemia de 
COVID-19 no âmbito da Atenção Primária à Saúde (APS) da Secretaria de Estado da Saúde do Distrito Federal (SES/ DF). Brasília: Secretaria de Estado de Saúde, 2020.

27. Araújo TM, Pinho PS, Almeida MMG. Prevalência de transtornos mentais comuns em mulheres e sua relação com as características sociodemográficas e o trabalho doméstico. Rev. Bras. Saude Mater. Infant. 2005;5(3):337-348.

28. Prado MAMB, Francisco PMSB, Barros MBA. Uso de medicamentos psicotrópicos em adultos e idosos residentes em Campinas, São Paulo : um estudo transversal de base populacional. Epidemiol. Serv. Saúde. 2017;26(4):747-758. DOI: $10.5123 / \mathrm{S} 1679-49742017000400007$

29. Senicato C, Lima MG, Barros, MBA. Ser trabalhadora remunerada ou dona de casa associa-se à qualidade de vida relacionada à saúde ? Cad. Saúde Pública. 2016;32(8):1-12. DOI: 10.1590/0102-311X00085415

30. Milos V, Rekman E, Eriksson T, Jakobsson U, Westerlund T, Midlo P. Improving the Quality of Pharmacotherapy in Elderly Primary Care Patients Through Medication Reviews : A Randomised Controlled Study. Drugs Aging. 2013;235-246. DOI: 10.1007/s40266-013-0057-0
31. Borges TL, Miasso AI, Vedana KGG, , Filho PCPT, Hegadoren KM. Prevalência do uso de psicotrópicos e fatores associados na atenção primária à saúde. Acta paul. enferm. 2015;28(4):344-349. DOI: 10.1590/19820194201500058

32. Wenceslau LD, Ortega F, Mental G. Saúde mental na atenção primária e Saúde Mental Global : perspectivas internacionais e cenário brasileiro. Interface (Botucatu). 2015;19(55):11211132. DOI: $10.1590 / 1807-57622014.1152$

33. BRASIL. Agência Nacional de Vigilância Sanitária. Resolução da Diretoria Colegiada (RDC) número 357 de 24 de março de 2020. Estende, temporariamente, as quantidades máximas de medicamentos sujeitos a controle especial permitidas em Notificações de Receita e Receitas de Controle Especial e permite, temporariamente, a entrega remota definida por programa público específico e a entrega em domicílio de medicamentos sujeitos a controle especial, em virtude da Emergência de Saúde Pública de Importância Internacional (ESPII) relacionada ao novo Coronavírus (SARS-CoV-2). Diário Oficial da União, Brasília, 24 de Março de 2020. 